\title{
ADUBAÇÃO COM TORTA DE MAMONA SOBRE O CRESCIMENTO E PRODUÇÃO DA AMOREIRA-PRETA
}

\author{
Letícia Vanni Ferreira $^{1 *}$, Carine Cocco $^{2}$, Daiana Finkenauer ${ }^{3}$, Luciano Picolotto ${ }^{4}$, Luis \\ Eduardo Corrêa Antunes ${ }^{5}$
}

\footnotetext{
${ }^{1}$ Eng. Agr. Dra ${ }^{a}$. Pós-doutoranda CAPES-EMPRAPA. Embrapa Clima Temperado, Pelotas, RS. *E-mail: letivf@hotmail.com

${ }^{2}$ Eng. Agr. Dr ${ }^{\mathrm{a}}$ Professora Universidade de Caxias do Sul, Caxias do Sul, RS

${ }^{3}$ Bióloga, MsC. Colégio Dinâmico, Chapecó, SC.

${ }^{4}$ Eng. Agr. Dr. Professor. Universidade Federal de Santa Catarina, Curitibanos, SC.

${ }^{5}$ Eng. Agr. Dr. Pesquisador. Embrapa Clima Temperado, Pelotas, RS.
}

RESUMO: A amoreira-preta é uma espécie rústica e, apesar de ter sido introduzida no Brasil na década de 70 , poucos trabalhos relacionados ao manejo fitotécnico foram realizados. Objetivou-se com a presente pesquisa avaliar o efeito vegetativo e produtivo em amoreira-preta, do uso de diferentes doses de torta de mamona como adubação de préplantio. O experimento a campo foi implantado em área experimental da Embrapa Clima Temperado, Pelotas, RS e, as análises químicas, no Laboratório de Nutrição Vegetal da mesma instituição, no período de 2008 a 2012. Os tratamentos foram: T1- 0; T2- 200; T3400; T4- 800; T5- $1.600 \mathrm{~g}$ de torta de mamona por planta, aplicados no momento da implantação do pomar. O delineamento experimental foi em blocos ao acaso. Avaliou-se a massa seca de poda de formação, o número de hastes, o comprimento das hastes, a massa seca de poda de produção, a produção, o número médio de frutas por planta, a massa média de frutas, o diâmetro das frutas e o conteúdo de sólidos solúveis. Em 2010 foi realizada análise do teor de nutrientes nas folhas, determinando-se os seguintes elementos: $\mathrm{N}, \mathrm{P}, \mathrm{K}$, $\mathrm{Ca}, \mathrm{Mg}, \mathrm{Fe}, \mathrm{Mn}, \mathrm{Zn}$ e $\mathrm{Cu}$. As variáveis avaliadas não foram influenciadas pelas doses de torta de mamona. Não foram verificados efeitos residuais de longo prazo sobre o desenvolvimento vegetativo, produtivo e dos macro e micronutrientes da amoreira-preta Tupy. Neste sentido, conclui-se que o uso de torta de mamona não causa efeito no comportamento da planta de amoreira-preta nas condições do presente trabalho.

Palavras-chave: Rubus. Amora-preta. Nutrição. Ricinus communis.

\section{FERTILIZATION OF PRE-PLANTING WITH CASTOR BEAN PIE IN THE DEVELOPMENT VEGETOPRODUTIVO OF BLACKBERRY}

\begin{abstract}
The blackberry tree is a rustic species and, despite having been introduced in Brazil in the 70s, few studies of this management were performed. The objective of this study was to evaluate the effect on vegetative and productive blackberry, using different doses of castor bean as a pre-planting fertilizer. The field experiment was conducted in an experimental field of Embrapa Temperate Agriculture, Pelotas/RS, and the chemical
\end{abstract}

Cultura Agronômica, Ilha Solteira, v.27, n.1, p.34-43, 2018 
analyzes, the Laboratory of Plant Nutrition of the same institution, in the period 2008-2012. The treatments were: T1-0; T2-200; T3-400; T4-800; T5-1600g of castor bean per plant. The experimental design was randomized blocks. The experimental design was randomized blocks. We evaluated the dry mass formation pruning, the number of stems, the length of the rods, dry mass production pruning, the yield, the average number of fruits per plant, average fruit weight, the diameter of fruit and soluble solids. In 2010 analysis of the nutrient content in leaves was performed by determining the following elements: $\mathrm{N}, \mathrm{P}, \mathrm{K}, \mathrm{Ca}, \mathrm{Mg}$, $\mathrm{Fe}, \mathrm{Mn}, \mathrm{Zn}$ and $\mathrm{Cu}$. The variables were not affected by the doses of castor bean, have not been verified residual long-term effects on the vegetative, productive and development of macro and micronutrients in shoots of the mulberry tree Tupy. In this sense it is concluded that the use of castor cake has not effect on the behavior of the blackberry plant under the conditions of this study.

Key words: Rubus. Blackberry. Nutrition. Ricinus communis.

\section{INTRODUÇÃO}

Nos últimos anos, tanto a produção quanto o consumo da amora-preta (Rubus spp.) está aumentando no Brasil, especialmente no Rio Grande do Sul, maior produtor nacional, onde estima-se que sejam produzidas 2.209 toneladas em 239 hectares (ANTUNES et al., 2010; ANTUNES et al., 2014). É considerada uma cultura de retorno rápido, sendo cultivada especialmente por pequenos agricultores familiares que buscam, a possibilidade de produzir de forma sustentável, sem o uso de agroquímicos em pequenas áreas (ANTUNES et al., 2010; ANTUNES et al., 2014). Seu desempenho produtivo depende, dentre outros fatores, da cultivar e do sistema de condução adotado (FERREIRA et al., 2016), sendo possível a obtenção de resultados, inclusive de produção, já ao final do primeiro ciclo da cultura (PEREIRA et al., 2015b).

Esta frutífera se adapta bem ao sistema de produção ecológico, o qual representa uma forte tendência frente à sociedade. No entanto, há carência de informações nas adequações do sistema de produção convencional para o orgânico, na maioria das espécies cultivadas (DALASTRA et al., 2009; CAMPAGNOLO et al., 2012), entre elas a amoreira-preta. Dentre as principais informações demandadas encontra-se a adubação, importante especialmente durante a fase inicial de desenvolvimento da planta (PEREIRA et al., 2015a). $\mathrm{O}$ nitrogênio $(\mathrm{N})$ é o elemento que a amoreira necessita em maior quantidade, sendo o principal responsável pelo crescimento, desenvolvimento e produção das plantas (NARAGUMA e CLARK, 1998; MOHADJER et al., 2001; STRIK, 2008). Dentre as fontes de $\mathrm{N}$ estão as orgânicas, as quais o disponibilizam lentamente para as plantas. Como um exemplo de fonte orgânica de nutrientes, principalmente de $\mathrm{N}$, destaca-se a torta de mamona que em todo o mundo, seu uso predominantemente tem sido como adubo orgânico (ZUCHI et al., 2007).

Cultura Agronômica, Ilha Solteira, v.27, n.1, p.34-43, 2018 
De acordo com Silva et al. (2012), é justificável o uso da torta de mamona, por se tratar de material com características benéficas ao solo e à nutrição das plantas, no entanto, faz-se necessário um estudo mais detalhado, a longo prazo, sobre os possíveis efeitos residuais de sua aplicação. Neste sentido, o objetivo do trabalho foi avaliar o efeito da adubação de base com torta de mamona no crescimento e produção de amoreira-preta.

\section{MATERIAL E MÉTODOS}

O experimento foi implantado em 2008 e conduzido em área experimental da Embrapa Clima Temperado, Pelotas, RS. Foram realizadas a análise do solo de pré-plantio e da torta de mamona antes da implantação do pomar (Tabela 1). A cultivar utilizada foi a Tupy. Aplicou-se e incorporou-se a torta de mamona, conforme os seguintes tratamentos: T1: 0; T2: $200 \mathrm{~g}\left(952,4 \mathrm{~kg} \mathrm{ha}^{-1}\right)$; T3: $400 \mathrm{~g}\left(2.000 \mathrm{~kg} \mathrm{ha}^{-1}\right)$; T4: $800 \mathrm{~g}\left(3.809,5 \mathrm{~kg} \mathrm{ha}^{-1}\right)$; T5: $1.600 \mathrm{~g}_{\text {planta }}{ }^{-1} \mathrm{de}$ torta de mamona $\left(7.619 \mathrm{~kg} \mathrm{ha}^{-1} \mathrm{de}\right.$ torta), aplicados no momento plantio, com espaçamento de $0,7 \mathrm{~m}$ entre plantas e 3,0m entre linhas, totalizando uma densidade de 4.761,9 plantas ha ${ }^{-1}$. Cada tratamento teve cinco repetições com cinco plantas por parcela.

Tabela 1. Análise do solo de pré-plantio e interpretação dos resultados de acordo com as classes de fertilidade (Manual de calagem e adubação para os Estados do Rio Grande do Sul e de Santa Catarina, 2016) em amostra de solo coletada em 2008 e 2011. Laboratório de Nutrição Vegetal. Embrapa Clima Temperado, Pelotas/RS, 2015.

\begin{tabular}{|c|c|c|c|c|c|c|c|c|c|c|}
\hline \multirow{3}{*}{ Solo } & \multirow{2}{*}{$\begin{array}{l}\mathrm{pH} \\
\text { água }\end{array}$} & \multirow{2}{*}{ SMP } & M.O. & Argila & $\mathrm{K}$ & $\mathrm{P}$ & $\mathrm{Na}$ & B & $\mathrm{Al}$ & CTC \\
\hline & & & \multicolumn{2}{|c|}{$\%$} & \multicolumn{3}{|c|}{$\mathrm{mg} / \mathrm{dm}^{3}$} & \multicolumn{3}{|c|}{$\mathrm{Cmolc} / \mathrm{dm}^{3}$} \\
\hline & 5,8 & 6,6 & 1,2 & 17,0 & 120,0 & 7,1 & 6,0 & 0,3 & 0,0 & 10,21 \\
\hline & M & M & B & A & A & B & & & & M \\
\hline \multirow[t]{4}{*}{ Torta } & $\mathbf{N}$ & $\mathbf{P}$ & $\mathbf{K}$ & $\mathbf{C a}$ & Mg & $\mathbf{F e}$ & Mn & $\mathbf{Z n}$ & $\mathbf{C u}$ & B \\
\hline & \multicolumn{4}{|c|}{$\%$} & & \multicolumn{5}{|c|}{$\mathrm{mg} \mathrm{Kg}^{-1}$} \\
\hline & 4,2 & 0,7 & 0,7 & 2,0 & 2,3 & 10,5 & 470,0 & 102,0 & 30,0 & 16,0 \\
\hline & $\mathrm{N}$ & M & M & M & M-A & $\mathrm{A}$ & A & A & A & \\
\hline
\end{tabular}

As variáveis avaliadas foram: massa seca de poda de formação $\left(\mathrm{g}_{\text {planta }}{ }^{-1}\right)$, número de hastes, comprimento das hastes $(\mathrm{cm})$, massa seca de hastes $\left(\mathrm{g} \mathrm{planta}^{-1}\right)$, produção por planta $\left(\mathrm{g}\right.$ planta $\left.^{-1}\right)$, número médio de frutas por planta, massa média de frutas $(\mathrm{g})$, diâmetro transversal e longitudinal das frutas $(\mathrm{mm})$ e sólidos solúveis ( ${ }^{\circ}$ Brix). Em junho de 2010 foi realizada análise do teor de nutrientes nas folhas, determinando-se os seguintes elementos: N, P, K, Ca, Mg, Fe, Mn, Zn e Cu. Antes do início da colheita de 2011, foi realizada uma análise química de solo, com três subamostras em cada unidade experimental, sendo determinados os teores de matéria orgânica, $\mathrm{pH}$ em água, índice $\mathrm{SMP}, \mathrm{Al}, \mathrm{P}, \mathrm{K}, \mathrm{Ca}, \mathrm{Mg}, \mathrm{Na}$, $\mathrm{S}, \mathrm{B}, \mathrm{Cu}, \mathrm{Fe}, \mathrm{Zn}, \mathrm{Mn}$.

O delineamento experimental adotado foi em cinco blocos ao acaso, com cinco doses de torta de mamona aplicadas no plantio. As avaliações foram realizadas nas safras 2009/2010 2010/2011 e 2011/2012. Os dados foram submetidos à análise de variância pelo 
teste F. Quando o efeito de tratamento foi significativo, realizou-se o teste de Tukey ao nível de 5\% de probabilidade de erro. As análises estatísticas foram realizadas com o emprego do programa SISVAR versão 5.1 (FERREIRA, 2011).

\section{RESULTADOS E DISCUSSÃO}

Não houve significância para o efeito de doses de torta de mamona em pré-plantio sobre a maioria das variáveis avaliadas, tanto nas avaliações vegetativas realizadas em 2009/2010 como para as variáveis produtivas das safras 2010/2011 e 2011/2012 (Tabela 2). Diferentemente dos resultados encontrados neste trabalho, a torta de mamona trouxe benefícios às algumas culturas como o morango. Vignolo et al. (2011) encontraram diferenças na produção de morangos adubados com diferentes doses torta de mamona, além de variar também o elemento $\mathrm{P}$ e $\mathrm{K}$, o que pode ter proporcionado esse aumento de produção.

As diferentes doses de torta de mamona fornecidas na adubação de amoreira-preta interferiram na massa seca de formação, sendo que quanto maior foi a dose aplicada maior foi o material retirado na poda (Tabela 1). Estes dados estão de acordo com Barreto et al., (2016), os quais verificaram que a adubação com torta de mamona influenciou no peso dos ramos de figueiras. Possivelmente, o nitrogênio presente em maio quantidade na maior dose de torta de mamona proporcionou maior crescimento vegetativo das plantas.

Acredita-se que a torta de mamona não apresente efeito residual de longo prazo, uma vez que a aplicação foi feita em pré-plantio e em todas as avaliações do experimento desde então, não observou-se efeito no desenvolvimento, tanto vegetativo quanto produtivo, apenas na fase inicial de desenvolvimento das plantas. Guertal (2009) indica que o uso de torta de mamona proporciona benefícios como redução da lixiviação de $\mathrm{N}$, o aumento da eficiência do uso do $\mathrm{N}$ e a redução dos custos de produção, embora não sejam amplamente demonstrados.

A massa seca de poda retirada na fase de formação das plantas foi afetada pelas doses de torta de mamona, modificando o desenvolvimento das hastes. Verificou-se um acréscimo da massa com a maior dose $\left(996,3 \mathrm{~g}\right.$ parcela $\left.^{-1}\right)$ se comparada à testemunha $(266,5$ g parcela ${ }^{1}$ ), acumulando nutrientes nas folhas e assim proporcionando um crescimento de hastes diferenciado. Porém, não houve diferença estatística para as variáveis número e comprimento médio das hastes, possivelmente pela baixa concentração de alguns nutrientes na folha.

A produção foi maior na safra de 2011/2012, provavelmente devido ao maior número de horas de frio acumuladas neste período e à chuva no período produtivo, a qual praticamente não ocorreu em 2010/2011, além das plantas estarem na idade de pico de produção. Este aumento também pode ser explicado, segundo Pereira et al. (2013) pelo maior desenvolvimento de órgãos perenes da planta, como sistema radicular e coroa.

Cultura Agronômica, Ilha Solteira, v.27, n.1, p.34-43, 2018 
Tabela 2. Massa seca de poda de formação (MSF) em g planta ${ }^{-1}$, número médio de hastes $(\mathrm{NMH})$, comprimento das hastes $(\mathrm{CH})$ em $\mathrm{cm}$, massa seca de poda de produção (MSP) em $\mathrm{g}$ fruta $^{-1}$, produção de frutas (PP) em g planta $^{-1}$, número médio de frutas (NMF), massa média de fruta (MMF) em $\mathrm{g}$ fruta $^{-1}$, diâmetro médio das frutas (DF) em $\mathrm{mm}$ e teor de sólidos solúveis (SS) submetidas a diferentes doses de torta de mamona em pré-plantio, nas safras de, 2009/2010, 2010/2011 e 2011/2012, amoreira-preta cv. Tupy. Embrapa Clima Temperado, Pelotas, RS, 2016.

\begin{tabular}{|c|c|c|c|c|c|c|c|c|c|}
\hline$\overline{\text { DTM }}$ & MSF & NMH & $\mathrm{CH}$ & MSP & $\mathbf{P P}$ & NMF & MMF & DF & SS \\
\hline \multicolumn{10}{|c|}{ 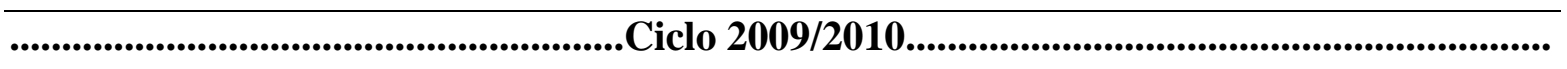 } \\
\hline 0 & $266,5 \mathrm{~b}$ & $5,8^{\mathrm{ns}}$ & $69,1^{\mathrm{ns}}$ & $2006,3^{\mathrm{ns}}$ & - & - & - & - & - \\
\hline 200 & $790,0 \mathrm{a}$ & 7,4 & 84,1 & 1583,3 & - & - & - & - & - \\
\hline 400 & $635,5 \mathrm{~b}$ & 7,5 & 76,2 & 2123,8 & - & - & - & - & - \\
\hline 800 & 762,4 al & $\mathrm{b} 8,3$ & 73,2 & 2703,7 & - & - & - & - & - \\
\hline 1600 & $996,3 \mathrm{a}$ & 6,9 & 72,3 & 2156,8 & - & - & - & - & - \\
\hline \multicolumn{2}{|c|}{ C.V.1 $^{1}(\%) 38,6$} & 24,4 & 11,3 & 29,59 & - & - & - & - & - \\
\hline \multicolumn{10}{|c|}{ 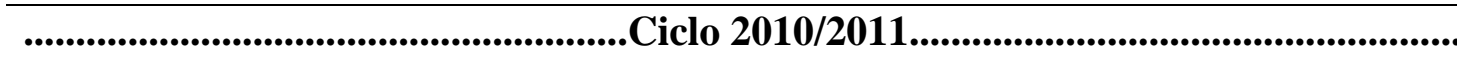 } \\
\hline $\mathbf{0}$ & - & - & - & - & $152,6^{\mathrm{ns}}$ & $189,7^{\mathrm{ns}}$ & $4,5^{\mathrm{ns}}$ & $27,0^{\mathrm{ns}}$ & - \\
\hline 200 & - & - & - & - & 972,1 & 220,2 & 4,5 & 25,8 & - \\
\hline 400 & - & - & - & - & 940,7 & 199,2 & 4,6 & 26,5 & - \\
\hline 800 & - & - & - & - & 912,8 & 263,6 & 3,7 & 27,0 & - \\
\hline 1600 & - & - & - & - & 871,9 & 181,9 & 4,8 & 24,4 & - \\
\hline C.V. ${ }^{1}(\%$ & & - & - & & 24,0 & 24,3 & 18,13 & 5,6 & - \\
\hline
\end{tabular}

\begin{tabular}{|c|c|c|c|c|c|c|c|c|c|}
\hline ...... & $\cdots$ & & & $\mathrm{Ci}$ & $11 / 2012 .$. & .................. & .............. & -10. & ............. \\
\hline $\mathbf{0}$ & - & - & - & - & $2053,2^{\mathrm{ns}}$ & $275,2^{\mathrm{ns}}$ & $7,4^{\mathrm{ns}}$ & - & $7,1^{\mathrm{ns}}$ \\
\hline 200 & - & - & - & - & 2787,7 & 362,1 & 7,7 & - & 8,1 \\
\hline 400 & - & - & - & - & 2487,9 & 323,6 & 7,7 & - & 7,2 \\
\hline 800 & - & - & - & - & 2499,5 & 330,3 & 7,6 & - & 8,2 \\
\hline 1600 & - & - & - & - & 1994,0 & 263,9 & 7,5 & - & 7,6 \\
\hline C.V.1 & & - & - & - & 22,6 & 22,1 & 5,9 & - & 19,4 \\
\hline
\end{tabular}

${ }^{1}$ C.V.(\%) Coeficiente de variação. *Médias seguidas por letras distintas, minúsculas na coluna, diferem entre si pelo Teste de Tukey a 5\% de probabilidade de erro. *ns (não significativo) a 5\% de probabilidade de erro.

Em 2010/2011, embora não havendo diferenças significativas para a massa média de fruta o maior valor observado foi nas frutas das plantas que receberam a maior dose de torta de mamona $\left(4,8 \mathrm{~g}_{\text {fruta }}{ }^{-1}\right)$ e o menor valor nas plantas que receberam a dose de $800 \mathrm{~g} \mathrm{planta}^{-}$ ${ }^{1}\left(3,7 \mathrm{~g} \mathrm{fruta}^{-1}\right)$ (23\% de diferença). Já na safra seguinte, os maiores valores observados de massa média por fruta foram nas plantas que receberam as doses 200 e $400 \mathrm{~g}$ planta $^{-1} \mathrm{de}^{\mathrm{d}}$ torta de mamona $\left(7,7 \mathrm{~g}_{\text {fruta }}{ }^{-1}\right)$. E o menor valor para esta variável foi observado nas plantas que receberam a dose $0\left(7,4 \mathrm{~g} \mathrm{fruta}^{-1}\right)$. É importante o equilíbrio na adubação, pois o tamanho das frutas está relacionado com a lei do mínimo. A análise mostrou que os nutrientes $\mathrm{N}$ e $\mathrm{K}$ encontraram-se abaixo do normal, o que provavelmente interferiu na massa média das frutas. 
Embora não tenha havido diferença estatística entre os tratamentos, observou-se que a maior dose de torta de mamona possibilitou menor quantidade de frutas, entretanto com maior massa média. Já na dose de $800 \mathrm{~g} \mathrm{planta}^{-1}$ o número de frutas produzidas foi maior, porém com menor massa (Tabela 2). Quando o $\mathrm{P}$ encontra-se insuficiente, como ocorre neste trabalho, a produção de frutas é pequena e de baixa qualidade (FREIRE, 2007; PEREIRA et al., 2012).

Em 2011/2012 resultados semelhantes foram obtidos. As plantas que receberam a dose de $800 \mathrm{~g} \mathrm{planta}^{-1}$ apresentaram o maior número médio de frutas, (330,3 frutas), seguido das plantas que receberam 200 e $400 \mathrm{~g}$ de torta de mamona por planta (362,1 e 323,6 respectivamente), sendo que o menor número de frutas foi observado nas plantas com $1600 \mathrm{~g}$ planta $^{-1}$ de torta de mamona (263,9 frutas), embora esta diferença não tenha sido significativa.

A variável sólidos solúveis (SS) não foi significativa para o fator doses de torta de mamona em pré-plantio. O valor médio de SS neste experimento foi de $7,6^{\circ}$ Brix na safra 2011/2012 (Tabela 2). Os resultados indicam que as adubações testadas não influenciam na concentração de SS das frutas, possivelmente estas doses não tenham sido suficientes para diferenciar a qualidade das amoras. Além disso, como não houve diferença no desenvolvimento vegetativo, as plantas receberam a mesma insolação, não modificando a concentração de SS nas frutas.

Pela análise de solo, para macro e micro elementos, não se verificou efeitos residuais significativos em função da adubação com torta de mamona nos teores de matéria orgânica, pH e índice SMP. O teor de matéria orgânica do solo não diferiu significativamente em nenhum dos fatores estudados, fato que favoreceu a padronização de características qualitativas dos frutos e produtiva das plantas. De acordo com as classes de fertilidade do solo, todos os tratamentos apresentaram valores classificados como baixo $(<2,5 \%)$, prejudicando assim a absorção e acúmulo no tecido das plantas.

Quanto ao $\mathrm{pH}$ e índice SMP do solo também não houve diferenças significativas entre os tratamentos no ano de 2011. Para o pH os tratamentos apresentaram valores entre 5,6-6,0, indicando serem índices ideais para o bom desenvolvimento da cultura (Tabela 1). Os teores de $\mathrm{P}, \mathrm{K}, \mathrm{Ca}$ e $\mathrm{Mg}$ também não foram influenciados pelo fator estudado, possivelmente pelo fato do $\mathrm{pH}$ não ter sido modificado entre os tratamentos o que favorece de forma igual a disponibilidade dos nutrientes absorvido pelas plantas.

De acordo com o Manual de calagem e adubação para os Estados do Rio Grande do Sul e de Santa Catarina, 2016, o teor de P nos tratamentos estava entre baixo a médio (14,121,0 $\mathrm{mg} \mathrm{dm}^{3}$ ), $\mathrm{K}$ médio (41-60 $\mathrm{mg} \mathrm{dm}^{3}$ ), Ca médio (2,1-4,0 cmolc dm³) e $\mathrm{Mg}$ de médio

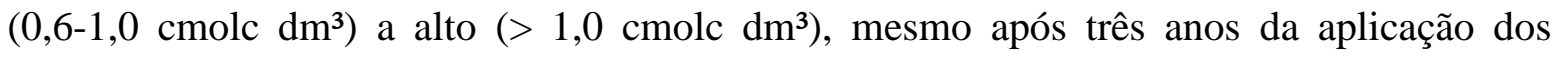
tratamentos com torta de mamona. $\mathrm{O}$ baixo teor de $\mathrm{P}$ era esperado, já que a quantidade do mesmo é baixa na torta de mamona, não sendo suficiente para elevar os teores deste nutriente, os quais já estavam baixos no momento da implantação do experimento. Então,

Cultura Agronômica, Ilha Solteira, v.27, n.1, p.34-43, 2018 
torna-se fundamental a adição de fontes alternativas deste mineral, especialmente quando se trabalha com sistemas ecológicos de produção.

Para os teores de micronutrientes no solo, em 2011, também não houve efeito significativo do fator estudado (Tabela 1). De acordo com o Manual de calagem e adubação para os Estados do Rio Grande do Sul e de Santa Catarina, 2016, os valores observados não foram influenciados pelas diferentes doses de adubação utilizadas e foram considerados altos: $\mathrm{Cu}\left(>0,4 \mathrm{mg} \mathrm{dm}^{3}\right), \mathrm{Fe}\left(>5,0 \mathrm{~g} \mathrm{dm}^{3}\right), \mathrm{Zn}\left(>0,5 \mathrm{mg} \mathrm{dm}^{3}\right)$ e $\mathrm{Mn}\left(>5,0 \mathrm{mg} \mathrm{dm}^{3}\right)$, possivelmente pelos valores do $\mathrm{pH}$ estarem nas faixas de maior disponibilidade de nutrientes.

A análise foliar das plantas revelou que a adubação com torta de mamona não apresentou efeito residual ao longo dos três anos avaliados para os teores de macro e micronutrientes nos tecidos vegetais (Tabela 3). De acordo com o Manual de calagem e adubação para os Estados do Rio Grande do Sul e de Santa Catarina (2016), o teor de Mn estava acima do normal (301-1000 mg kg-1), Zn normal (15-50 mg kg-1), Cu normal (6-25 $\left.\mathrm{mg} \mathrm{kg}{ }^{-1}\right)$, Ca normal (0,6-2,5 \%), Mg normal (0,3-1,0\%), P insuficiente $(<0,2 \%)$ devido a baixa quantidade no solo e na torta de mamona. Nos tratamentos sem torta de mamona e com dose máxima de torta os valores médios de $\mathrm{N}$ mostraram-se abaixo do normal $(1,75$ $2,19 \%)$, já nos demais tratamentos mostraram-se dentro da normalidade $(2,20-3,00 \%)$.

Tabela 3. Teores de macronutrientes, teores de matéria orgânica (M.O), pH, índice SMP e teores de micronutrientes no solo e teores de macro e micronutrientes em folhas de amoreira-preta 'Tupy" sob diferentes doses de torta de mamona (DTM), no ano de 2011. Embrapa Clima Temperado, Pelotas/RS, 2016.

\begin{tabular}{|c|c|c|c|c|c|c|c|c|c|c|c|}
\hline DTM & $\mathbf{P}^{1}$ & $\mathbf{K}^{1}$ & $\mathbf{C a}^{2}$ & $\mathrm{Mg}^{2}$ & M.O & pH & SMP & $\mathrm{Cu}^{3}$ & $\mathrm{Zn}^{3}$ & Mn & $\mathrm{Fe}^{3}$ \\
\hline 0 & $11,8^{\mathrm{ns}}$ & $59,4^{\mathrm{ns}}$ & $2,3^{\mathrm{ns}}$ & $1,0^{\mathrm{ns}}$ & $1,1^{\mathrm{ns}}$ & $5,8^{\mathrm{ns}}$ & $6,4^{\mathrm{ns}}$ & $2,0^{\mathrm{ns}}$ & $2,9^{\mathrm{ns}}$ & $12,0^{\mathrm{ns}}$ & $3,0^{\mathrm{ns}}$ \\
\hline 200 & 11,9 & 54,2 & 2,4 & 0,9 & 1,0 & 6,0 & 6,5 & 1,6 & 2,4 & 10,7 & 3,1 \\
\hline 400 & 12,4 & 54,2 & 2,3 & 0,9 & 1,3 & 5,6 & 6,4 & 1,6 & 2,8 & 13,2 & 3,0 \\
\hline 800 & 15,8 & 54,8 & 2,7 & 1,1 & 1,2 & 6,0 & 6,5 & 1,5 & 3,1 & 9,7 & 3,3 \\
\hline 1600 & 15,2 & 50,6 & 2,6 & 1,0 & 1,3 & 5,9 & 6,4 & 1,5 & 2,7 & 9,9 & 2,7 \\
\hline$\overline{\text { C.V.1 }}(\%)$ & 40,1 & 23,4 & 14,4 & 15,5 & 21,6 & 5,5 & 3,1 & 44,2 & 30,4 & 28,3 & 7,2 \\
\hline \multirow[t]{2}{*}{ DTM } & $\mathbf{N}$ & $\mathbf{P}$ & $\mathbf{K}$ & $\mathbf{C a}$ & & Mg & $\mathbf{F e}$ & & Mn & Zn & $\mathrm{Cu}$ \\
\hline & \multicolumn{6}{|c|}{$\%$} & \multicolumn{5}{|c|}{$\mathrm{mg} \mathrm{kg}^{-1}$} \\
\hline 0 & $2,1^{\mathrm{ns}}$ & $0,16^{\mathrm{ns}}$ & $1,2^{\mathrm{ns}}$ & $0,7^{\mathrm{ns}}$ & & $0,3^{\mathrm{ns}}$ & $155,8^{\mathrm{ns}}$ & & $6,8^{\mathrm{ns}}$ & $27,6^{\mathrm{ns}}$ & $8,0^{\mathrm{ns}}$ \\
\hline 200 & 2,2 & 0,18 & 1,2 & 0,7 & & 0,3 & 183,8 & & 8,4 & 29,0 & 8,6 \\
\hline 400 & 2,3 & 0,18 & 1,3 & 0,7 & & 0,3 & 179,0 & & 3,4 & 30,0 & 9,2 \\
\hline 800 & 2,2 & 0,18 & 1,2 & 0,7 & & 0,3 & 186,0 & & 7,6 & 30,4 & 9,4 \\
\hline 1600 & 2,2 & 0,19 & 1,2 & 0,7 & & 0,3 & 169,4 & & 2,6 & 27,0 & 8,4 \\
\hline$\overline{\text { C.V.1(\%) }}$ & 7,2 & 12,9 & 4,3 & 5,2 & 4,8 & 19,1 & & & 9,8 & 11,8 & 12,7 \\
\hline
\end{tabular}

${ }^{1}$ C.V.(\%) Coeficiente de variação. *ns (não significativo) a 5\% de probabilidade de erro.

Os teores de $\mathrm{K}$ apresentaram-se abaixo do normal (1,00-1,24\%) nos tratamentos que receberam $1600 \mathrm{~g}_{\text {planta }^{-1}}$ de torta de mamona e no tratamento testemunha, já nos outros Cultura Agronômica, Ilha Solteira, v.27, n.1, p.34-43, 2018 
tratamentos apresentou-se normal (1,25-3,00\%). É possível que a torta de mamona apresente liberação rápida. As doses testadas podem ter sido ser muito baixas para que a planta pudesse expressar um incremento significativo na produção de frutas. Este fato vem a confirmar a ausência de efeito significativo da adubação com torta de mamona no presente trabalho nas variáveis produtivas da planta. Pereira et al. (2013) relata que a exportação de nutrientes varia em função da cultivar e da produtividade, sendo que observou exportação na 'Tupy' de 1,71 t de P por tonelada de fruta, 6,84 t de K por t de fruta e 1,60 $\mathrm{t}$ de Ca por $\mathrm{t}$ de fruta, já 'Xavante' exportou 1,53 t de P por tonelada de fruta, 5,38 t de K por t de fruta e 1,93 t de Ca por tonelada de fruta.

Durante os ciclos de produção avaliados, não foram feitas adubações complementares, objetivando-se avaliar o efeito residual. Entretanto a ausência de diferença significativa pode ser devido a este fato, ou seja, a aplicação de torta de mamona ter sido realizada apenas em 2008, na implantação do pomar. Possivelmente, aplicações com intervalos menores favoreceria a absorção pela planta, portanto, novas verificações neste sentido são importantes.

\section{CONCLUSÃO}

As diferentes doses de torta de mamona testadas não interferiram no desenvolvimento vegetativo e na produção da amoreira-preta.

\section{AGRADECIMENTOS}

À Coordenação de Aperfeiçoamento de Pessoal de Nível Superior (CAPES) e à Empresa Brasileira de Pesquisa Agropecuária (EMBRAPA) pelo apoio financeiro.

\section{REFERÊNCIAS BIBLIOGRÁFICAS}

ANTUNES. L. E. C.; GONÇALVES, E. D.; TREVISAN, R. Fenologia e produção de cultivares de amoreira-preta em sistema agroecológico. Ciência Rural, Santa Maria, v. 40, n. 9, p.1929-1933, 2010. Disponível em: <http://dx.doi.org/10.1590/S010384782010000900012>. Acesso em: 10 fev. 2007.

ANTUNES, L. E. C.; PEREIRA, I. S.; VIGNOLO, G. K.; GONÇALVES, M. A. Produção de amoreira-preta no Brasil. Revista Brasileira de Fruticultura, Jaboticabal, v. 36, n. 1, p.100-111, 2014. Disponível em: <http://dx.doi.org/10.1590/0100-2945-450/13>. Acesso em: 22 fev. 2016.

BARRETO, C. F.; LUCIO, P.; MARTINS, C. R. Efeito da torta de mamona e tungue como fertilizante orgânico e manejo de irrigação na produção da figueira em sistema orgânico. Revista Congrega Urcamp, Bagé, v. 13, n. 1, p. 48, 2016. CD-ROM 
CAMPAGNOLO, M. A.; PIO, R. Produção da amoreira-preta 'Tupy' sob diferentes épocas de poda. Ciência Rural, Santa Maria, v. 42, n. 2, p.225-231, 2012. Disponível em <http://dx.doi.org/10.1590/S0103-8478201200500000.7>. Acesso em: 02 fev. 2013.

DALASTRA, I. M.; PIO, R.; CAMPAGNOLO, M. A.; DALASTRA, G. M.; CHAGAS, E. A.; GUIMARÃES, V. F. Época de poda na produção de figos verdes 'Roxo de Valinhos' em sistema orgânico nas regiões oeste do Paraná. Revista Brasileira de Fruticultura, Jaboticabal, v. 31, n. 2, p.447-453, 2009. Disponível em: <http://www.scielo.br/pdf/rbf/v31n2/v31n2a19.pdf>. Acesso em: 16 set. 2011.

FERREIRA, D. F. Sisvar: a computer statistical analysis system. Ciência e Agrotecnologia, Lavras, v. 35, n. 6, p.1039-1042, 2011.

FERREIRA, L. V.; PICOLOTTO, L.; COCCO, C.; FINKENAUER, D.; ANTUNES, L. E. C. Produção de amoreira-preta sob diferentes sistemas de condução. Ciência Rural, Santa Maria, v. 46, n. 3, 2016. Disponível em: 〈http://dx.doi.org/10.1590/0103-8478cr20140601〉. Acesso em: 22 fev. 2016.

FREIRE, C. J. S. Nutrição e adubação. In: ANTUNES, L. E. C.; RASEIRA, M. C. B. (Eds.). Cultivo da amoreira-preta. Pelotas: Embrapa Clima Temperado, 2007. cap. 5, p. 45-54. (Sistemas de Produção, 12).

GUERTAL, E. A. Slow-release nitrogen fertilizers in vegetable production: A review. Hort Technology, Alexandria, v. 19, n. 1, p.16-19, 2009.

MANUAL de calagem e adubação para os Estados do Rio Grande do Sul e de Santa Catarina. 11.ed. Porto Alegre: Sociedade Brasileira de Ciência do Solo, Núcleo Regional Sul, Comissão de Química e Fertilidade do Solo, 2016. 376 p.

MOHADJER, P.; STRIK, B. C.; ZEBARTH, J. RIGHETTI, T. L. Nitrogen uptake, partitioning and remobilization in 'Kotata' blackberry in alternate-year production. Journal of Horticultural Science and Biotechnology, Reino Unido, v. 76, n. 6, p.700-708, 2001.

NARAGUMA, J.; CLARK, J. R. Effect of nitrogen fertilization on 'Arapaho' thornless blackberry. Communications in Soil Science and Plant Analysis, Marília, v. 29, n. 1, p.2775-2783, 1998.

PEREIRA, I. S.; ANTUNES, L. E. C.; MESSIAS, R. S.; SILVEIRA, C. A. P.; VIGNOLO, G. K. Avaliações da subtração dos elementos N, P e K sobre a produção e qualidade de frutos de amoreira-preta. Pelotas: Embrapa Clima Temperado, 2012. (Comunicado Técnico, 275). Disponível em: <http://ainfo. cnptia.embrapa.br/digital/bitstream/item/61569/1/Comunicado-275.pdf $>$. Acesso em: 20 jun. 2014.

PEREIRA, I. S.; ANTUNES, L. E. C.; MESSIAS, R. S.; SILVEIRA, C. A. P.; VIGNOLO. G. Adubação nitrogenada e características agronômica sem amoreira-preta. Pesquisa Agropecuária Brasileira, Brasília, v. 48, n. 4, p.373-380, 2013. Disponível em <http://www.scielo.br/pdf/pab/v48n4/04.pdf>. Acesso em 10 jan. 2014.

Cultura Agronômica, Ilha Solteira, v.27, n.1, p.34-43, 2018 
PEREIRA, I. S.; PICOLOTTO, L.; GONÇALVES, M. A.; ANTUNES, L. E. C. Potassium Fertilization Affects Floricane Mineral Nutrient Content, Growth, and Yield of Blackberry. HortScience, Alexandria, v. 50, n. 8, p.1234-1240, 2015. (a)

PEREIRA, I. S.; NAVA, G.; PICOLOTTO, L. VIGNOLO, G. K.; GONÇALVE, M. A.; ANTUNES, L. E. C. Exigência nutricional e adubação da amoreira-preta. Revista Ciências Agrárias, Lisboa, v. 58, n. 1, p.96-104, 2015. (b) Disponível em <http://dx.doi.org/10.4322/rca.1755>. Acesso em: 22 fev. 2016.

SILVA, S. D.; PRESOTTO, R. A.; MAROTA, H. B.; ZONTA, E. Uso de torta de mamona como fertilizante orgânico. Pesquisa Agropecuária Tropical, Goiânia, v. 42, n. 1, p.19-27, 2012. Disponível em: <http://www.agro.ufg.br/pat>. Acesso em: 20 set. 2017.

STRIK, B. C. A review of nitrogen nutrition of Rubus. Acta Horticulturae, Pucón, v. 777, n. 1, p.403-410, 2008.

VIGNOLO, G. K.; ARAÚJO, V. F.; KUNDE, R. J.; SILVEIRA, C. A. P.; ANTUNES, L. E. C. Produção de morangos a partir de fertilizantes alternativos em pré-plantio. Ciência Rural, Santa Maria, v. 41, n. 10, p.1755-1761, 2011. Disponível em <http://www.scielo.br/pdf/cr/v41n10/a12711cr5238>. Acesso em: 20 abr. 2012.

ZUCHI, J.; BEVILAGUA, G. P.; GALHO, A. M.; MARQUES, R. L. L. Efeito de torta de mamona sobre componentes de rendimento de trigo. Revista Brasileira de Agroecologia, Guarapari, v. 2, n. 2, p.1080-1083, 2007. 